Case Report

\title{
Successful Technique for Closure of Macular Hole Retinal Detachment Using Autologous Retinal Transplant
}

\author{
Juan Abel Ramirez-Estudillo $\mathbb{D}^{\mathbb{D}}{ }^{1}$ Geovanni Rios-Nequis $\mathbb{D}^{1},{ }^{1}$ Martin Jimenez-Rodríguez $\mathbb{D}^{1}{ }^{1}$ \\ Hugo Valdez-Flores $\mathbb{D}^{1},{ }^{1}$ and Ximena Ramirez-Galicia $\mathbb{D i}^{2}$ \\ ${ }^{1}$ Retina Department, Fundacion Hospital Nuestra Señora De La Luz IAP, Mexico \\ ${ }^{2}$ Research Department, Faculty of Medicine, Universidad La Salle, Mexico \\ Correspondence should be addressed to Martin Jimenez-Rodríguez; martinretina@icloud.com
}

Received 20 May 2020; Revised 12 October 2020; Accepted 23 October 2020; Published 19 November 2020

Academic Editor: Claudio Campa

Copyright (c) 2020 Juan Abel Ramirez-Estudillo et al. This is an open access article distributed under the Creative Commons Attribution License, which permits unrestricted use, distribution, and reproduction in any medium, provided the original work is properly cited.

\begin{abstract}
Macular hole retinal detachment (MHRD) for the most part develops in highly myopic eyes. Several surgical methods have been introduced to treat MHRD. We describe our experience with the autologous retinal transplant in patient with MHRD. A 49-yearold female presented with a 2-week history of a sudden decrease in the central vision in the right eye (RE). A 3-port, 25-gauge pars plana vitrectomy was performed with the ILM dye staining and peeling. Endodiathermy was applied around a 1.5-disc diameter neurosensory donor site in the supertemporal retina. The graft was cut with standard 25-gauge curved scissors. Perfluoro-n-octane (PFO) was instilled. The free graft was gently handled until its packing into the macular hole. Two months following the initial PPV, the macular hole was closed, and vision improved from 0.05 to $0.25 \operatorname{logMAR}$.
\end{abstract}

\section{Introduction}

Macular hole retinal detachment (MHRD) mostly develops in highly myopic eyes. Since Gonvers and Machemer reported using pars plana vitrectomy (PPV) and gas tamponade in 1982 [1], several surgical methods have been introduced to treat MHRD, such as macular buckling, vitrectomy, and gas or silicon oil tamponade fluid with internal limiting membrane (ILM), inverted ILM flap technique, and scleral imbrication [1-6]. In recent years, the autologous neurosensory retinal transplant has been considered like a surgical technique in the treatment for closure of the refractory macular hole. We describe our case with the autologous retinal transplant in patient with MHRD.

\section{Case Presentation}

A 49-year-old female presented with a 2-week history of a sudden decrease in the central vision in the right eye (RE). The patient had no significant history of systemic disease. At her initial visit, the best corrected visual acuity (BCVA) was $0.05 \operatorname{logMAR}$ in her RE and $1.00 \log \mathrm{MAR}$ in her left eye. The slit-lamp examination of the anterior segment and intraocular pressure were normal, and the lens status was phakic of both eyes. A fundus examination revealed macular hole retinal detachment in her RE, lattice degeneration, and atrophic holes were found.

The surgery was performed under general anaesthesia, and we elected to use no. 41 circumferential scleral buckle owing to the fact that another predisposal lesions were found 


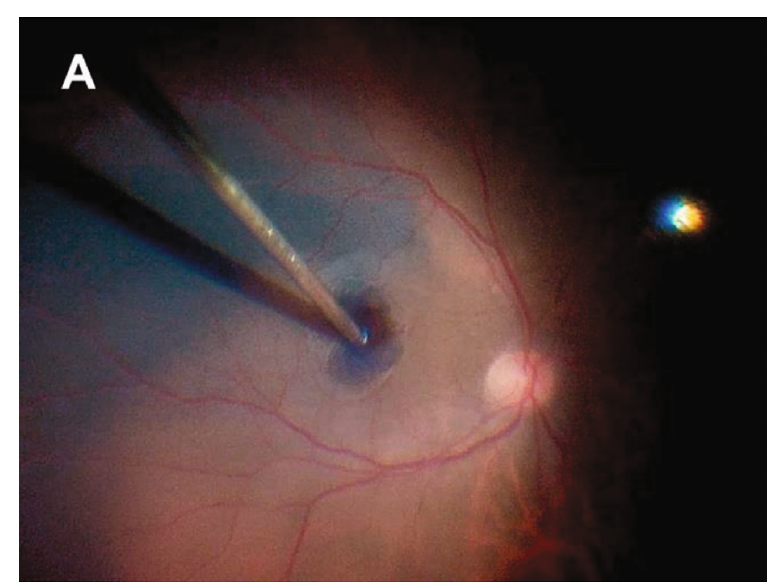

(a)

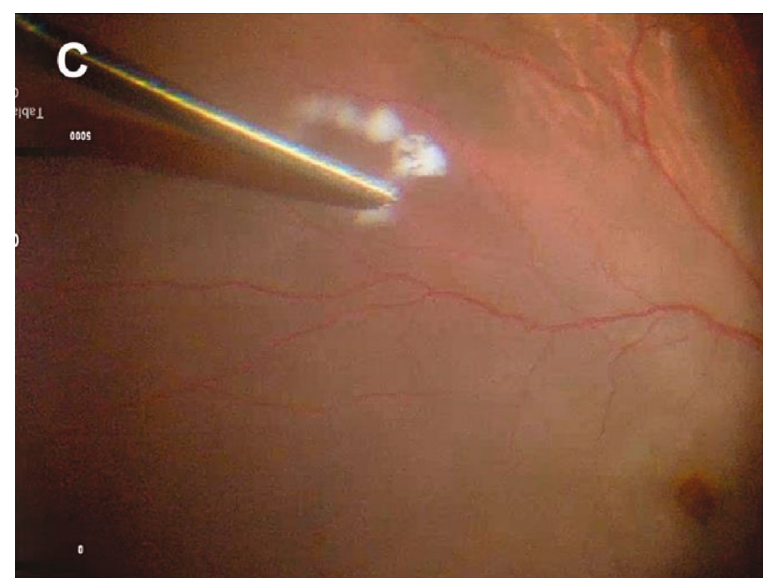

(c)

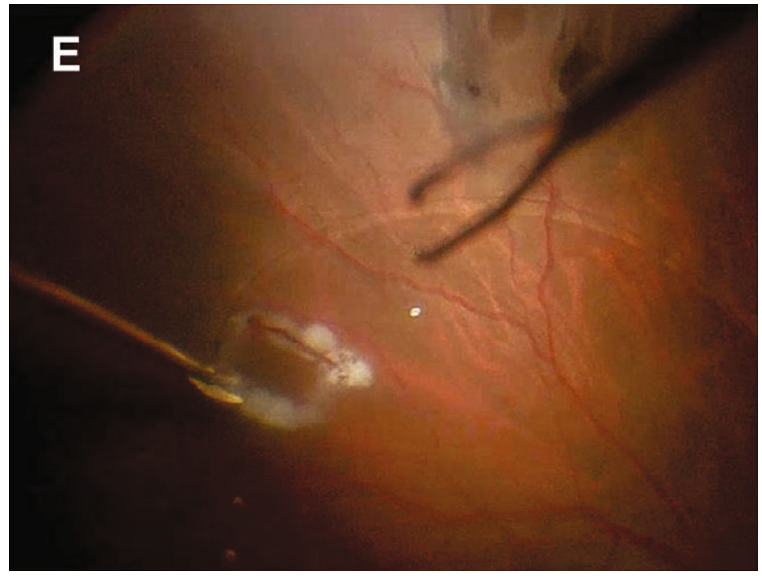

(e)

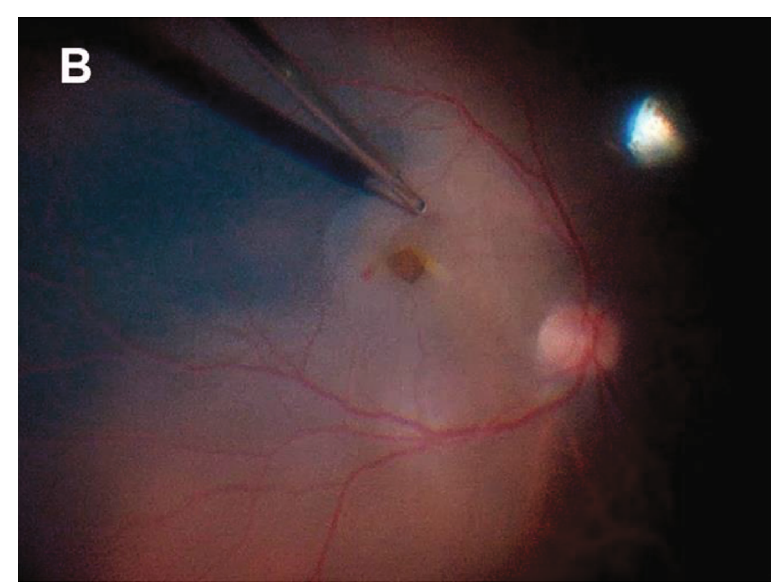

(b)

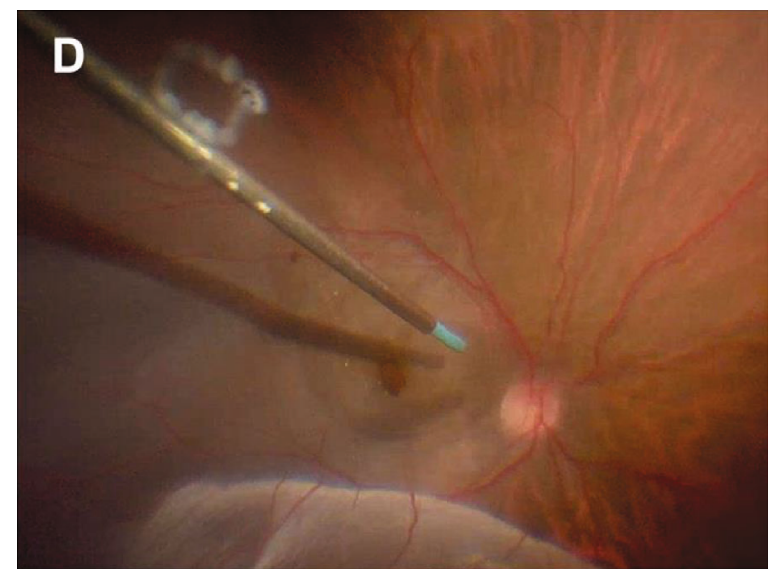

(d)

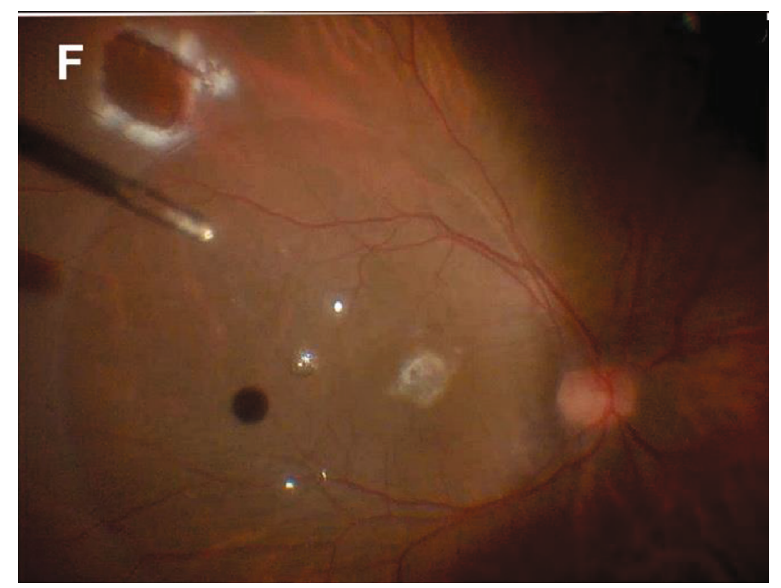

(f)

Figure 1: Continued. 


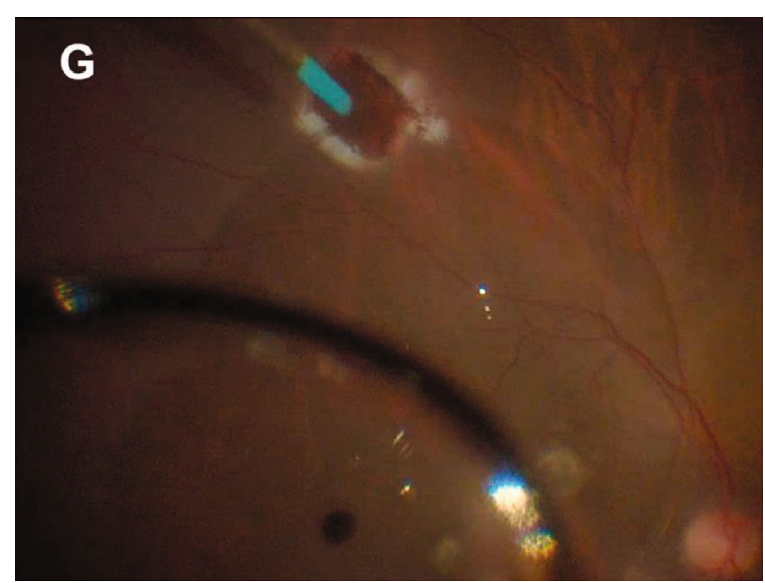

(g)

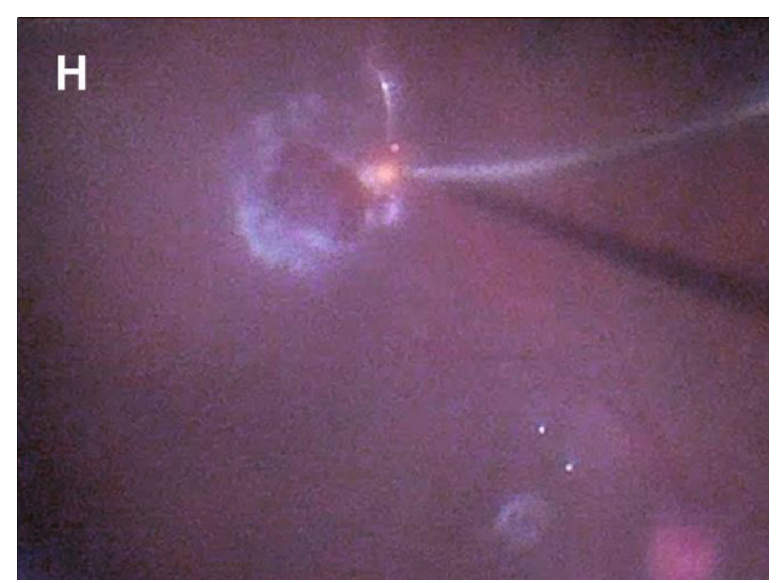

(h)

FIGURE 1: (a) Intraoperative digital photography showing subretinal migration of brilliant blue during dye staining. (b) The internal limiting membrane has been removed and is grasped with the vitreous forceps. Notice the absence of blue coloration at the macular area at the region of internal limiting membrane ablation. (c) Endodiathermy was applied around a 1.5-disc diameter neurosensory retina. (d) Injection of the perfluoro-n-octane in the retinal interface. (e) The graft cuts were linear, being a square graft. (f) Image showing free graft packing inside the macular hole. (g) A fluid air exchange was performed, and subretinal fluid was extracted through the area where the graft was taken. (h) Laser around the area where the graft was taken.

in the inferior retina. A standard vitrectomy equipment (Constellation, Alcon Surgical, Ft. Worth, TX), Lumera 700 microscope (Carl Zeiss Germany) and NGENUITY 3D visualization system (Alcon Surgical, Ft. Worth, TX) were used. A 3-port, 25-gauge PPV was performed; after posterior hyaloid detachment, the ILM was dyed (TissueBlue, DORC). The ILM peeling was performed on the detached retina in a circular fashion, pulling off approximately 1.0 disk diameters of ILM centered on the foveal defect. We should mention that subretinal migration of brilliant blue was detected; opportunely, when the dye was aspirated, part of the subretinal fluid came out at the same time with the dye (Figure 1). Peripheral vitrectomy with indentation was done before taking the graft.

Endodiathermy was applied around a 1.5-disc diameter neurosensory donor site. The harvest location was selected in the more bullous zone of the retina (superior and temporal) in order to avoid touching the choroid. The graft was cut with standard 25-gauge curved scissors to approximately $90 \%$ of its final size to keep the tissue stable.

Perfluoro-n-octane (PFO) was instilled to completely cover the donor site, the free graft was teared and manipulated into the correct position using end grasping forceps, and the tissue was gently handled until packing it into the macular hole.

A fluid air exchange was performed, and subretinal fluid was extracted through the area where the graft was taken. Laser endophotocoagulation was applied as retinopexy in the donor site and the predisposal lesions, and 5000 centistoke silicone oil was used as tamponade to the finish the surgery.

One day after the surgery, the retinal was completely attached, and the free graft stayed inside in the macular hole.
Two months following the initial PPV, the macular hole was closed, and the optical coherent tomography (OCT) showed integration of the retinal flap and partial restoration of the internal plexiform layer (Figure 2). Best corrected visual acuity improved to $0.25 \log$ MAR at 2 months of the surgery.

\section{Discussion}

The coexistent macular hole was described in approximately $1 \%$ to $4 \%$ of cases of rhegmatogenous retinal detachment. According to the pathological mechanisms, several surgical methods have been introduced to the treatment and the retinal reattachment rate ranged from $40 \%$ to $90 \%$ [7-10], but the macular hole closure rate was lower, approximately $40 \%$ (range 35\%-70\%) [10]. In 2015, the technique involving the use of an autologous neurosensory retinal free flap for closure of the refractory myopic macular hole was described $(\mathrm{MH})$ [11]; since then, several series have been published that mentions the complete anatomical closure of $\mathrm{MH}$ by OCT can reach up to $87.8 \%$.

In this case, single surgery achieved the retina reattachment and $\mathrm{MH}$ closure. Although this type or surgery has been referred [12], the technique with detached retina has not described formerly. The presence of retinal detachment was not a problem for the management of free graft of the neurosensory retina; in fact, in our opinion, the subretinal fluid due to retinal detachment facilitated the harvest by maintaining the neurosensory retina separate from the retinal pigment epithelium and helps avoiding touching the choroid instead of developing an hydraulic retinal detachment with the aid of a 38 or $41 \mathrm{G}$ syringe that can cause subretinal hemorrhage under the graft or subretinal blood migration 


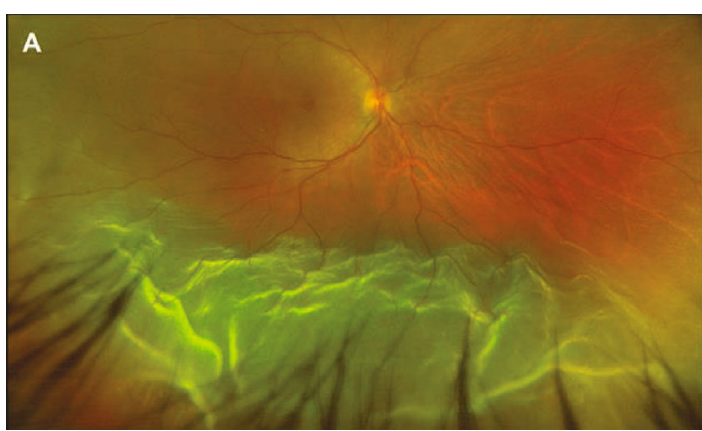

(a)

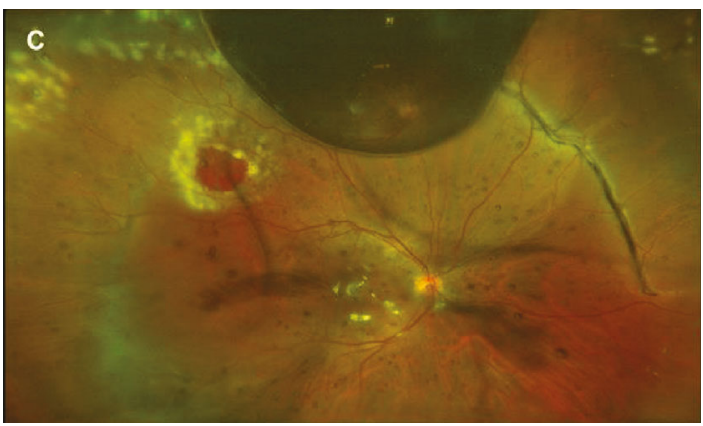

(c)

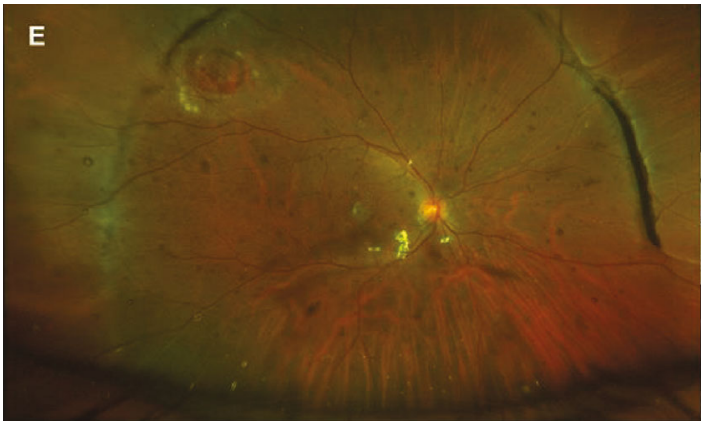

(e)

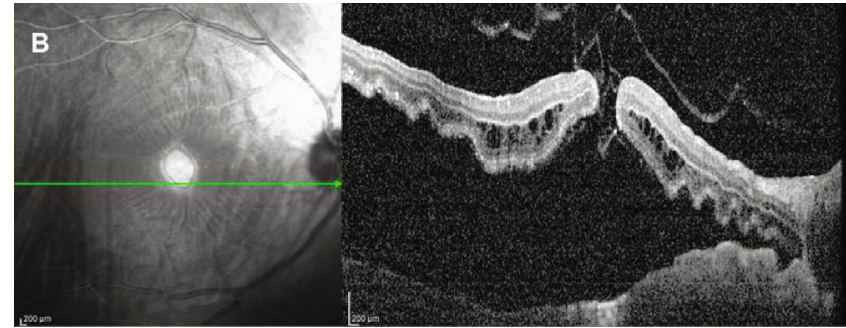

(b)

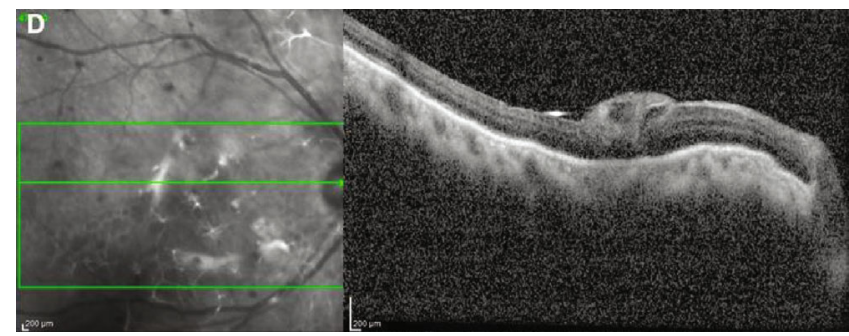

(d)

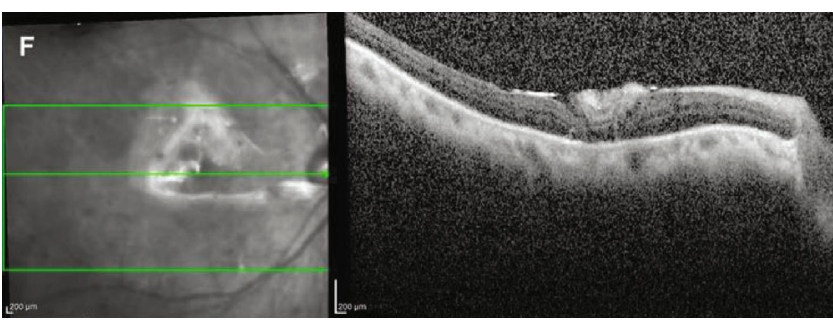

(f)

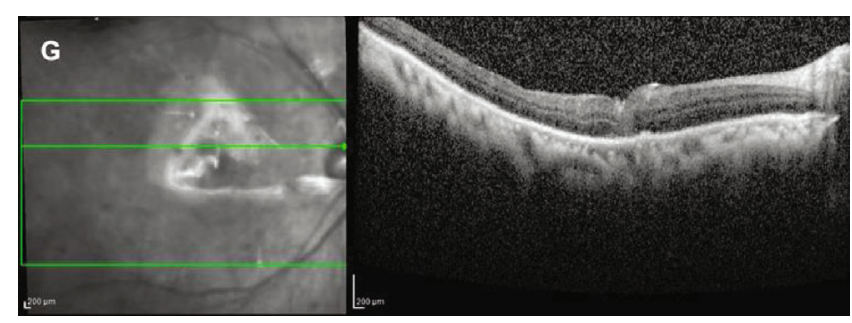

(g)

FIgURE 2: (a) Fundus photo RE shows macular hole retinal detachment. (b) Preoperative optical coherence tomography (OCT) demonstrates macular detachment with full-thickness macular hole. (c) One day after surgery, the foveal contour had improved by the free graft. (d) OCT postop 1 day and the hyperreflective retinal flap are visualized in place over the $\mathrm{MH}$. (e) Color photograph of the fundus of the right eye two months after the autologous retina transplant shows retina reattachment. (f) One month postoperatively, there was significant integration of the retinal flap with improved architecture of the inner retinal layers, and some hyperreflective lines show the graft.(g) At 2 months postoperatively, there was significant integration of the retinal flap.

causing toxicity. 5000 centistoke silicon oil was used because the patient was not able to stay in the city; aside, it allowed us to keep up the graft by OCT image with better quality than SF6 bubble.

\section{Conclusions}

According to the evolution of this case, we believe it is possible to initiate a series of patients with this technique 
in order to consider autologous retinal transplant as a first therapeutic possibility in the retinal detachment associated with a macular hole.

\section{Data Availability}

The data used to support the findings of this study are included within the article.

\section{Disclosure}

The research did not receive any specific funding, although the Hospital Nuestra Señora de la Luz allowed its facilities for exploration, surgery, and patient monitoring.

\section{Conflicts of Interest}

The authors declare no conflict of interest in the aims for this article.

\section{References}

[1] M. Gonvers and R. Machemer, "A new approach to treating retinal detachment with macular hole," American Journal of Ophthalmology, vol. 94, no. 4, pp. 468-472, 1982.

[2] F. Ando, "Use of a special macular explant in surgery for retinal detachment with macular hole," Japanese Journal of Ophthalmology, vol. 24, pp. 29-34, 1980.

[3] E. H. Ryan, C. T. Bramante, R. A. Mittra et al., "Management of rhegmatogenous retinal detachment with coexistent macular hole in the era of internal limiting membrane peeling," American Journal of Ophthalmology, vol. 152, no. 5, pp. 815819.e1, 2011.

[4] Z. Michalewska, J. Michalewski, R. A. Adelman, and J. Nawrocki, "Inverted internal limiting membrane flap technique for large macular holes," Ophthalmology, vol. 117, no. 10, pp. 2018-2025, 2010.

[5] E. Ortisi, T. Avitabile, and V. Bonfiglio, "Surgical management of retinal detachment because of macular hole in highly myopic eyes," Retina, vol. 32, no. 9, pp. 1704-1718, 2012.

[6] Z. Hu, X. Gu, H. Qian et al., "Perfluorocarbon liquid-assisted inverted limiting membrane flap technique combined with subretinal fluid drainage for macular hole retinal detachment in highly myopic eyes," Retina, vol. 10, no. 2, pp. 140-144, 2016.

[7] C.-C. Lai, Y.-P. Chen, N.-K. Wang et al., "Vitrectomy with internal limiting membrane repositioning and autologous blood for macular hole retinal detachment in highly myopic eyes," Ophthalmology, vol. 122, no. 9, pp. 1889-1898, 2015.

[8] X. Li, W. Wang, S. Tang, and J. Zhao, "Gas injection versus vitrectomy with gas for treating retinal detachment owing to macular hole in high myopes," Ophthalmology, vol. 116, no. 6, pp. 1182-1187.e1, 2009.

[9] L. S. Lim, A. Tsai, D. Wong et al., "Prognostic factor analysis of vitrectomy for retinal detachment associated with myopic macular holes," Ophthalmology, vol. 121, no. 1, pp. 305-310, 2014.

[10] F. U. M. I. T. A. K. A. ANDO, N. O. R. I. O. OHBA, K. O. U. TOUURA, and H. I. R. O. S. H. I. HIROSE, "ANATOMICAL AND VISUAL OUTCOMES AFTER EPISCLERAL MACULAR BUCKLING COMPARED WITH THOSE AFTER PARS
PLANA VITRECTOMY FOR RETINAL DETACHMENT CAUSED BY MACULAR HOLE IN HIGHLY MYOPIC EYES," Retina, vol. 27, no. 1, pp. 37-44, 2007.

[11] D. S. Grewal and T. H. Mahmoud, "Autologous Neurosensory Retinal Free Flap for Closure of Refractory Myopic Macular Holes," JAMA Ophthalmology, vol. 134, no. 2, pp. 229-230, 2016.

[12] D. S. Grewal, S. Charles, B. Parolini, K. Kadonosono, and T. H. Mahmoud, "Autologous retinal transplant for refractory macular holes: multicenter international collaborative study group," Ophthalmology, vol. 126, no. 10, pp. 1399-1408, 2019. 